\title{
The importance of clinical picture in the diagnosis of Guillain-Barré syndrome
}

\author{
Tudorita-Gabriela Paranga ${ }^{2}$, Maria Obreja' ${ }^{1}$, Claudia-Elena Plesca ${ }^{1,2}$, \\ Daniela Leca ${ }^{1,2}$, Larisa Miftode ${ }^{1,2}$, Oana Stamateanu ${ }^{1,2}$, Elisabeta Lupu ${ }^{3}$, \\ Egidia-Gabriela Miftode ${ }^{1,2}$ \\ 1"Gr. T. Popa" University of Medicine and Pharmacy, Iasi, Romania \\ 2"St. Parascheva" Infectious Disease Clinical Hospital, lasi, Romania \\ ${ }^{3}$ Regional Institute of Oncology, Iasi, Romania
}

\begin{abstract}
Guillain-Barré syndrome (GBS) is an immune-mediated peripheral polyradiculonevritis, which is clinically characterized by rapid, symmetrical progression of muscle weakness or paralysis, with or without sensitive symptoms.

We report the case of a male child aged 4 years is presented for distal weakness in the lower limbs, impossibility of maintaining vertical posture, balance disorders, alternating sleepiness with episodes of psychomotor agitation and dysphonia, headache, with sudden onset about 12 hours before hospitalization.

The presented case underlines the importance of accurately describing the clinical picture and evolution in establishing the diagnosis, in total or partial absence of paraclinical data. Also, the low number of cases described in medical jurnals justify the interest in investigating and publishing these cases.
\end{abstract}

Keywords: Guillain-Barré syndrome, polyradiculonevritis, muscle weakness or paralysis, clinical picture, paraclinical data

\section{INTRODUCTION}

Guillain-Barré syndrome (GBS) is an immunemediated peripheral polyradiculonevritis, which is clinically characterized by rapid, symmetrical progression of muscle weakness or paralysis, with or without sensitive symptoms, the description of which has been made for the first time in the year 1916 (1). Diagnosis of the disease is a clinical one, supported by CSF results and motor conduction studies. Early recognition of the disease and prompt therapeutic intervention can shorten the period of the disease, reduce the severity and minimize the need for pulmonary ventilation (2).

\section{CASE PRESENTATION}

We report the case of a male child aged 4 years is presented for distal weakness in the lower limbs, impossibility of maintaining vertical posture, balance disorders, alternating sleepiness with episodes of psychomotor agitation and dysphonia, headache, with sudden onset about 12 hours before hospitalization. The patient had a recent history of acute otitis media, treated with Cefuroxime, for 7 days, and an episode of respiratory infection with laryngeal phenomena.

The clinical exam revealed a moderately influenced general condition, with preserved conscious- 
ness, palmar and facial erythrosis, mild pharyngeal congestion, dehydrated lips, ocular and nasal catarrh, muscular hypotonia, balance marked disorders, impossibility of walking and maintaining vertical posture, extinguished hoarse voice, bilateral basal harsh vesicular murmur, rhythmic heart sounds, without extra sounds, painless abdomen, intestinal transit and miction in physiological conditions, without signs of meningeal tightness. The suspicion of polyradiculonevritis was raised and serological testing for enteroviruses, Mycoplasma pneumoniae, Epstein-Barr virus, stool sampling for enteroviruses, tonsillar exudate and stool sampling for Campylobacter were performed. In view of a possible upper / otic respiratory tract infection, therapy with ceftriaxone associated with dexamethasone was initiated.

On the 3rd day of admission, on the physical exam there is a progression of neurological phenomena with abolition of osteo-tendinous reflexes, without impaired sensitivity, accentuated dysphonia and difficulty in swallowing solid food. The lumbar puncture was performed, revealing the absence of inflammatory reaction and hyperalbuminorachia $(0.56 \mathrm{~g} / 1$ with reference values $0-0.35 \mathrm{~g} / \mathrm{l})$. A thoracic radiography is conducted, describing bilateral pulmonary interstitial infiltration, while cerebral MRI emphasizes changes in acute otomastoiditis, clinical elements requiring the reshuffle of initial antibiotherapy with meropenem and vancomycin. The neurological exam performed on day 4 highlights difficulty in ingesting liquids and solids, ortostatism and walking are not possible, head movements cannot be controlled, sitting position is maintained with difficulty and not without support, active segmented movements are diminished as strength and speed at upper limb level and absent in the lower limbs, muscular hypotonia predominantly of the lower limbs, stylo radial and tricipital reflexes are present, the patellar reflex is absent bilaterally, the superficial abdominal reflexes are present symmetrically, bilateral plantar reflexes, cremasteric reflex absent bilaterally, absence of coordination in the lower limbs, upper limbs do not respond, seemingly without any disorders regarding sensitivity, language or psychic.

The initial diagnosis suspicion is maintained and it is decided upon the initiation of therapy with human Immunoglobulins IV (iv IG) 5 grams per day, rehydration infusions, group B vitamins and clarithromycin in oral administration (targeting atypical germs). The neurological revaluation performed at 10 hours reveals the following: fluid and solid dysphagia (tracheal aspiration procedure is performed and insertion of naso-gastric feeding tube is recommended), dysarthria with nasal voice and hypotonia of neck muscles, sore muscles upon compression, slowed intestinal transit, and the tendency to retract the bilateral Achilles tendon. In the following days, the serious general condition of the child is maintained, with subfebrile episodes, while the neurological phenomena ascend to the upper limbs, the active movements of which are limited, uncoordinated and accompanied by paresthesia.

For objective reasons, plasmapheresis could not be initiated and therapy was continued with immunoglobulin increased to 6.5 grams.

The subsequent evolution was relatively favourable, with the slow remission of neurological phenomena. Neurological recovery continues, and on the 18th day of hospitalization muscle strength is found to have improved (the patient maintains prehension capacity for a few seconds), in assisted sitting position the patient is able to hold his head, he mobilizes the lower limbs from the hip joint and has attempts to lift them on the bed plane. When discharged (day 20), the child can sit alone at the edge of the bed for 1-2 minutes, clap and sing. The peculiarity of the case resides in a very rapid neurological deterioration with ascending Landry syndrome, but with full recovery at one-month evaluation after discharge.

\section{DISCUSSIONS}

More than a century later from the first case described, the diagnosis and management of a GBS case remains a challenge in the current clinical practice. The annual incidence of this pathology is 1.5 cases/100,000, the susceptibility in men is greater than in women, and the incidence increases with age (3). With the eradication of poliomyelitis, GBS remains the most common cause of acute motor paralysis in children. The syndrome more often affects children aged between 1 and 5 years, predominantly of the male sex (4). While some studies show an incidence in children between 0.34 and 
1.34/100,000 (5), a recent study from 2019 argues that rarely does a case occur under the age of 2 , while in children the incidence is $0.6 / 100,000$ (3).

As far as etiology is concerned, studies have shown that a proportion of approximately $70 \%$ of cases were preceded by acute infectious episodes 1-3 weeks before the onset of the symptomatology, most commonly viral episodes, usually respiratory or gastrointestinal (6).

There is an entire series of infectious agents that have been quoted in literature as trigger factors for GBS. Among them are cytomegalovirus, Campylobacter jejuni, Epstein-Barr virus, measles virus, influenza A virus, enterovirus D68, Mycoplasma pneumoniae and Zikka virus (3). Campylobacter jejuni infection has been reported more frequently in Asian countries and has been associated in a proportion of $25-50 \%$ with cases of GBS in adults.

In another study quoted in literature, Nasiri $\mathrm{J}$ et al. list the HIV virus among the infectious agents correlated with the onset of GBS. There have been mentioned in literature cases of GBS post-vaccination against influenza, poliomyelitis, rabies, and meningitis (8), but in a smaller percentage than those occurring in the context of those diseases.

In the case presented it was considered as a possible trigger factor a previous infection with the Epstein-Barr virus, enteroviruses or Mycoplasma pneumoniae, for which biological samples were taken. The anamnesis ruled out a digestive infection in recent history. IgM antibodies for Mycoplasma pneumoniae were present (>95 IU $/ \mathrm{ml}$, positive $>22 \mathrm{IU} / \mathrm{ml}$ ), indicating a recent infection. IgM and IgG serology for enteroviruses was negative. The diagnosis of Guillain-Barré syndrome is established on anamnestic, clinical and paraclinical criteria.

On the occasion of the possible association of GBS with a vaccination campaign against swine flu in America from 2009/2010, Brighton Collaboration developed a set of diagnostic criteria, which set the degree of diagnostic certainty based on signs found at clinical examination and additional tests, starting from the diagnostic level 1 with the highest degree of certainty and reaching level 4 that sums up reported cases as GBS, but with the absence of complete data necessary for a further classification (Table 1) (9).
TABLE 1. Diagnosis of Guillain-Barré syndrome and validation of Brighton criteria (9)

\begin{tabular}{|c|c|c|c|c|}
\hline \multirow[t]{2}{*}{ Diagnostic criteria } & \multicolumn{4}{|c|}{$\begin{array}{c}\text { Level of diagnostic } \\
\text { certainty }\end{array}$} \\
\hline & 1 & 2 & 3 & 4 \\
\hline Bilateral and flaccid weakness of limbs & + & + & + & +1 \\
\hline $\begin{array}{l}\text { Decreased or absent deep tendon } \\
\text { reflexes in weak limb }\end{array}$ & + & + & + & $+/$ \\
\hline $\begin{array}{l}\text { Monophasic course and time between } \\
\text { onset-nadir } 12 \mathrm{~h} \text { to } 28 \text { days }\end{array}$ & + & + & + & $+/$ \\
\hline CSF cell count $50 / \mu \mathrm{l}$ & + & $+\underline{a}$ & - & $+/$ \\
\hline $\begin{array}{l}\text { CSF protein concentration }>\text { normal } \\
\text { value }\end{array}$ & + & $+/-\underline{a}$ & - & $+/$ \\
\hline $\begin{array}{l}\text { NCS findings consistent with one of the } \\
\text { subtypes of GBS }\end{array}$ & + & $+/-$ & - & +1 \\
\hline $\begin{array}{l}\text { Absence of alternative diagnosis for } \\
\text { weakness }\end{array}$ & + & + & + & + \\
\hline
\end{tabular}

+ present,- absent, +/- present or absent;

NCS= nerve conduction; GBS= Guillain-Barré syndrome;

a If NCS is not collected or results not available nerve electrophysiology results must be consistent with the diagnosis Guillain-Barré syndrome

In the case described we have positive anamnesis for a history of respiratory infection. Clinically, the described picture is consistent with the Brighton criteria (table 1). Of the abovementioned criteria, the following have been identified in our case: flaccid muscular paralysis of the limbs, abolished osteo-tendinous reflexes in the affected limbs, the patient had a favourable monophasic evolution, although initially slower, and the period from the onset of the disease to discharge was 21 days. Also, from a paraclinical point of view, the patient presented the cytoalbuminologic dissociation (absence of inflammatory reaction in CSF with an increased albuminorachia), which coincides with one of the Brighton criteria. There are several studies mentioning that cytoalbuminologic dissociation in CSF is often absent in the early stages of the disease. Uncini et al. state that in the early days of disease the CSF proteins can be normal in $50 \%$ of cases (10).

However, our case presented this dissociation in the sample taken on the third day of illness. Nerve conduction and electromyography studies have not been performed. According to Brighton criteria, the case presented has a diagnostic certainty score of 2 .

Christiaan Fokke et al. conclude in a study that patients in a hierarchy of levels of diagnostic certainty according to the Brighton criteria do not differ in terms of clinical severity or evolution. There are studies in literature conducted on groups of patients distributed predominantly in levels 3 and 4 of 
diagnostic certainty according to these criteria, because in certain units, the necessary investigations are not performed or the results were unavailable, highlighting that clear and thorough documentation of clinical signs should allow a better classification of GBS in order for the diagnosis to be possible in both well-developed and developing countries (9).

This study also mentions that in countries such as the Netherlands the studies on neuronal electrophysiology and CSF analysis are not practiced when there is a high clinical suspicion of GBS and there are no clinical criteria for another disease, which strengthens the importance of clinical examination in such patients.

Various studies claim that neuronal electrophysiology may have a prognostic value but at present there are no well-defined and approved criteria of electrophysiological diagnosis of GBS, all currently described criteria focussing on subtype differentiation, the value of which is uncertain in current clinical practice (9).

Intravenously immunoglobulins (ivIG) or plasmapheresis with good supportive care is considered as treatment of choice by many studies. Both treatments are considered equally $\mathrm{V}$ effective, but there are authors claiming that ivIG are cheaper due to a shorter patient hospitalization (13). ivIG in the dose of $400 \mathrm{mg} / \mathrm{kg} /$ day for 5 days is recommended.

With regard to the treatment of the case described, it was chosen to initiate immunoglobulin IV therapy, administered starting from the 5th day of the child's admission. Plasmapheresis was not possible so the dose of immunoglobulin was increased.

The reason why in our case it was considered appropriate to increase the dose of immunoglobulin as an alternative to the absence of plasmapheresis is supported by the literature data $(11,12)$ which report the correlation of a slower evolution and a less favourable prognosis with those cases where the concentration of immune globulin in the serum does not reach the expected values (without a clear motivation) (11). In addition, there are data from literature claiming that iv IG is preferred in young children when plasmapheresis is technically difficult to proceed (14).

Evolution was slowly favourable. Comparative studies conducted on adults and children diagnosed with GBS reveal a better therapeutic response when children are concerned (6). The patient did not require intensive care measures.

Although progress in the field is evident, GBS has a mortality rate which varies between 3 and $7 \%$ in Europe and North America, even when using plasmapheresis and immunoglobulins, the best therapies currently existing (10).

\section{CONCLUSIONS}

The presented case underlines the importance of accurately describing the clinical picture and evolution in establishing the diagnosis, in total or partial absence of paraclinical data. Data in the literature regarding the low incidence of this pathology, especially in children, the fact that the diagnosis is established clinically, without biomarkers, according to not very clearly defined and unanimously accepted criteria in a disease with a heterogeneous clinical picture, justify the interest of the clinicians, and implicitly ours, in investigating and publishing these cases in order to draw the attention of young physicians on the need for rapid initiation of the diagnostic algorithm and appropriate therapy even in the absence of paraclinical explorations.

Conflict of interest: none declared Financial support: none declared

\section{REFERENCES}

1. Jin J, Hu F, Qin X, Liu X, Li M, Dang Y, Dang J. Very early neurophysiological study in Guillain-Barré syndrome. Eur Neurol 2018; 80(1-2):100-105.

2. Roodbol J, de Wit MY, van den Berg B, Kahlmann V, Drenthen J, Catsman-Berrevoets CE, Jacobs BC. Diagnosis of Guillain-Barré syndrome in children and validation of the Brighton criteria. $J$ Neurol 2017; 264(5):856-861.

3. Wachira VK, Peixoto HM, de Oliveira MRF. Systematic review of factors associated with the development of Guillain-Barre syndrome 2007-2017: What has changed? Trop Med Int Health 2019; 24(2):132-142.
4. Sejvar JJ, Baughman AL, Wise M, Morgan OW. Population incidence of Guillain-Barré syndrome: A systematic review and meta-analysis. Neuroepidemiology 2011; 36(2):123-133.

5. Sadek AA, Abou-Taleb A, Ali WA. Outcome of Guillain-Barré syndrome in children: A prospective cohort study in a tertiary hospital in Upper Egypt. Electron Physician 2016; 8(12):3318-3324.

6. Kumar M, Aroor S, Mundkur S, Kumar S. Guillain-barré syndrome: A clinical study of twenty children. J Clin Diagn Res 2015; 9(1):SC09-12.

7. Nasiri J, Ghazavi M, Yaghini O, Chaldavi M. Clinical features and outcome of Guillain-Barré syndrome in children. Iran J Child Neurol 2018; 12(2):49-57. 
8. Wajih Ullah M, Qaseem A, Amray A. Post vaccination Guillain-Barré syndrome: A case report. Cureus 2018; 10(4):e2511.

9. Fokke C, van den Berg B, Drenthen J, Walgaard C, van Doorn PA, Jacobs BC. Diagnosis of Guillain-Barré syndrome and validation of Brighton criteria. Brain 2014; 137(1):33-43.

10. Uncini A. Guillain-Barré syndrome: What have we learnt during one century? A personal historical perspective. Rev Neurol (Paris) 2016; 172(10):632-644.

11. Van Doorn PA, Kuitwaard K, Walgaard C, van Koningsveld R, Ruts L, Jacobs BC. IVIG treatment and prognosis in Guillain-Barré syndrome. J Clin Immunol 2010; 30(S1):74-78.
12. Kuitwaard K, de Gelder J, Tio-Gillen AP, Hop WC, van Gelder T, van Toorenenbergen AW, van Doorn PA, Jacobs BC. Pharmacokinetics of intravenous immunoglobulin and outcome in Guillain-Barré syndrome. Ann Neurol 2009; 66(5):597-603.

13. McGrogan A, Madle GC, Seaman HE, de Vries CS. The epidemiology of Guillain-Barré syndrome worldwide. A systematic literature review. Neuroepidemiology 2009; 32(2):150-163.

14. Jasti AK, Selmi C, Sarmiento-Monroy JC, Vega DA, Anaya JM, Gershwin ME. Guillain-Barré syndrome: Causes, immunopathogenic mechanisms and treatment. Expert Rev Clin Immunol 2016; 12(11):1175-1189. 